\title{
Einige kritische Anmerkungen zum geplanten Schweizer Finanzdienstleistungsgesetz
}

\author{
Sethe, Rolf
}

Posted at the Zurich Open Repository and Archive, University of Zurich

ZORA URL: https://doi.org/10.5167/uzh-132742

Book Section

Originally published at:

Sethe, Rolf (2016). Einige kritische Anmerkungen zum geplanten Schweizer Finanzdienstleistungsgesetz. In: Casper, Matthias; Klöhn, Lars; Roth, Wulf-Henning; Schmiers, Christian. Festschrift für Johannes Köndgen. München: RWS Verlag Kommunikationsforum GmbH, 599-614. 


\title{
Einige kritische Anmerkungen zum geplanten Schweizer Finanzdienstleistungsgesetz
}

\author{
ROLF SETHE
}

Inhaltsübersicht

I. Einleitung

II. Gründe für die aufsichtsrechtliche Neuregelung

III. Der Geltungsbereich der Wohlverhaltensregeln

1. Persönlicher Geltungsbereich

2. Sachlicher Geltungsbereich
3. Räumlicher Geltungsbereich

IV. Die Kundenkategorien

V. Die Interessenwahrungspflicht

1. Eignungs- und Angemessenheitstest

2. Organisatorische Pflichten zur

VI. Fazit

\section{Einleitung}

In Anlehnung an die bekannte Einleitung der Asterix-Hefte lässt sich die Situation der Schweiz folgendermaßen beschreiben:

„Wir befinden uns im Jahre 2015 n. Chr. Europa ist von der Europäischen Union dominiert [...] Ganz Europa? Nein! Ein unbeugsames Bergvolk leistet beharrlich Widerstand.“

Die Schweiz hatte den EWR-Beitritt am 6. Dezember 1992 abgelehnt. Die politische Diskussion um das Verhältnis zur Europäischen Union wird seitdem in zahlreichen Gremien und mit allerlei Erfolgen, Misserfolgen und überraschenden Wendungen fortgeführt. Ein Zaubertrank, mit dem man das gegenseitige Verhältnis zur Zufriedenheit aller politischen Akteure in der Schweiz und der Europäischen Union regeln könnte, ist nicht in Sicht, obwohl sich die „Druiden“ in Bern und Brüssel redlich bemühen. Dieses tagespolitische Geschäft muss uns hier nicht weiter interessieren. Aus Sicht des Bank- und Kapitalmarktrechtlers spannend sind die Gemeinsamkeiten und Unterschiede der aufsichtsrechtlichen Erfassung der Branche. Betrachtet man dieses Rechtsgebiet aus der Vogelperspektive, kann man eine Zweiteilung feststellen. ${ }^{1)}$

1) Hierzu etwa Heinemann, Rechtliche Transplantate zwischen Europäischer Union und der Schweiz, in: Fahrländer/Heizmann (Hrsg.), Europäisierung der schweizerischen Rechtsordnung, Zürich, St. Gallen, 2013, S. 3 ff.; Sethe, Rechtsangleichung im Schweizer Finanzmarktrecht - Heteronomer und autonomer Nachvollzug, Äquivalenz, Swiss Finish, in: FS für Höland, 2015, S. 345 ff.; Weber/Schaller, Auswirkungen der EU-Finanzmarktregulierung auf die schweizerische Finanzmarktgesetzgebung, EuZ 2004, 74 ff. 
Im Bereich des Bank- und des Börsenrechts ist die Schweiz in zahlreichen Gremien aktiv, die sich mit der Entwicklung internationaler Standards befassen, wie etwa das Basel Committee on Banking Supervision, der Financial Stability Board (FSB) oder die International Organization of Securities Commissions (IOSCO). Um wettbewerbsfähig zu bleiben, übernimmt sie regelmäßig internationale Regulierungsstandards, wie etwa die Basler Eigenkapitalvorschriften, die Vorgaben zum Derivatehandel aufgrund der G20-Beschlüsse von Pittsburgh oder die Regelungen zur Bekämpfung der Geldwäsche. In vielen Bereichen hat sie zudem freiwillig die europäischen Regelungen umgesetzt (sog. autonomer Nachvollzug), wie etwa im Verbraucherkreditrecht.

Auffällig ist, dass von dieser Entwicklung bislang zwei Bereiche ausgeklammert blieben, nämlich die Aufsicht über Anlageberater und Vermögensverwalter und die Verhaltens- und Organisationspflichten im Wertpapiervertrieb. Die Schweiz ist nun dabei, ihr Kapitalmarktrecht umzugestalten, wobei die genannten Bereiche neu in einem Finanzinstitutsgesetz (FINIG) und in einem Finanzdienstleistungsgesetz (FIDLEG) geregelt werden sollen. ${ }^{2}$ Aus Platzgründen kann ich in diesem Beitrag, mit dem ich Johannes Köndgen für die sehr bereichernde Zusammenarbeit als Mitherausgeber der ZBB danken möchte, nur auf einige wenige neuralgische Aspekte des geplanten FIDLEG eingehen. Dieses Thema findet hoffentlich das Interesse des Jubilars, der vor seinem Wechsel an die Universität Bonn im Jahre 1995 Ordinarius für Privatrecht, Handelsrecht und Rechtsvergleichung an der Hochschule St. Gallen war und daher das Schweizer Finanzmarktrecht von innen kennt.

\section{Gründe für die aufsichtsrechtliche Neuregelung}

Das schweizerische Finanzmarktrecht kennt im Gegensatz zur MiFID I bislang kein sektorübergreifendes Aufsichtsrecht für Finanzintermediäre, sondern hat in verschiedenen Einzelgesetzen (Teil-)Aspekte geregelt: So sind die Verhaltenspflichten zwischen dem Dienstleister und seinen Kunden - im Vergleich zur europäischen Regelung - sehr knapp in Art. 11 BEHG für Effektenhändler und im Investmentrecht gemäß Art. $20 \mathrm{ff}$. KAG für die Investmentbranche geregelt. Zwar finden sich daneben auf

2) Https://www.sif.admin.ch/sif/de/home/dokumentation/finweb/regulierungsprojekte/ finanzdienstleistungsgesetz--fidleg-.html. 
der Stufe der Selbstregulierung zahlreiche Erlasse, mit denen einzelne Finanzdienstleistungen vertieft geregelt werden, wie die „Verhaltensregeln für Effektenhändler bei der Durchführung des Effektenhandelsgeschäftes“ von 2008 oder die „Richtlinien für Vermögensverwaltungsaufträge“ von 2013. Vorgaben für die Anlagevermittlung, Abschlussvermittlung oder Anlageberatung durch zugelassene Intermediäre oder durch nicht zulassungspflichtige Finanzdienstleister fehlen dagegen. Das geplante FIDLEG soll eine sektorübergreifende Regelung schaffen und einheitliche aufsichtsrechtliche Verhaltens- und Organisationspflichten aufstellen.

Für die Schweiz ist eine derartige Regelung aus zahlreichen Gründen von großer Bedeutung. ${ }^{3)}$ Der international bedeutende Finanzplatz Schweiz passt sich aufgrund der globalen Verflechtung an internationale Standards an. Die rechtlichen Rahmenbedingungen sind ein entscheidender Faktor zum Erhalt der eigenen Wettbewerbsfähigkeit. Große Institute haben daher freiwillig die Verhaltenspflichten der Europäischen Union übernommen. Kleinere Institute und selbstständige Finanzintermediäre haben dies aus Kostengründen bislang gescheut.

Da die Schweizer Finanzintermediäre jedoch einen erheblichen Teil ihrer Finanzdienstleistungen in der Europäischen Union erbringen, sind sie auf den Zugang zum europäischen Markt angewiesen. Dieser Marktzugang ist nun gefährdet, da MiFID II/MiFIR ein neues Drittstaatenregime vorsehen. Die Europäische Union überlässt erstmals die Kompetenz, Instituten aus Drittstaaten den EU-Zugang zu gewähren, nicht mehr den Mitgliedstaaten. Will ein Finanzdienstleister aus einem Drittstaat seine Leistungen an bestimmte Kundengruppen (geeignete Gegenparteien und geborene professionelle Kunden) in der Europäischen Union erbringen, erhält er künftig den Marktzutritt nur, wenn im Drittland die effektive Gleichwertigkeit der Aufsichts- und Wohlverhaltensregeln gewährleistet ist (Art. 46 Abs. 2 lit. a, Art. 47 Abs. 1 MiFIR). ${ }^{4}$ Dieses Äquivalenzerfordernis zwingt die Schweiz damit indirekt zur weitgehenden Übernahme der MiFID II/ MiFIR-Regelungen. Sollen Leistungen gegenüber Retailkunden (Privatkunden und gekorene professionelle Kunden) erbracht werden, sind nach wie vor die Mitgliedstaaten befugt, die Anforderungen an den Drittstaaten-

3) Ausführlich dazu Sethe, MiFID II - Eine Herausforderung für den Finanzplatz Schweiz, SJZ 2014, $477 \mathrm{ff}$.

4) Ausführlich zum Drittstaatenregime Arquint, Internationalisierung der Finanzmarktaufsicht, GesKR 2014, 131, 139 f.; Sethe, Das Drittstaatenregime von MiFIR und MiFID II, SZW 2014, 615 ff. 
zugang zu regeln. Sie können wählen, ob sie die Errichtung einer Zweigstelle verlangen oder nicht. Tun sie dies, sind sie in den Anforderungen jedoch nicht mehr frei, sondern an die Vorgaben der Art. 39 ff. MiFID II gebunden. Eine der Voraussetzungen ist, dass die Drittlandfirma in ihrem Heimatstaat ordnungsgemäß errichtet und für die Dienstleistungen, für die sie eine Zulassung im Mitgliedstaat beantragt, in ihrem Heimatland zugelassen und beaufsichtigt ist (Art. 39 Abs. 2 lit. a MiFID II). Diese Voraussetzung bereitet der Schweiz im Bereich der prudentiellen Aufsicht über Banken und Effektenhändler keine Probleme, da ihr Aufsichtsrecht dem der Europäischen Union insoweit gleichwertig sein dürfte. Problematisch ist jedoch, dass eine prudentielle Aufsicht über unabhängige Vermögensverwalter und Anlageberater fehlt. Eine Zulassung von Zweigstellen dieser Finanzintermediäre in Mitgliedstaaten der Europäischen Union scheidet daher aus, solange sie in der Schweiz nicht beaufsichtigt sind.

Heikel ist weiterhin, dass das schweizerische Aufsichtsrecht bislang keine detaillierte Regelung der Verhaltens- und Organisationspflichten beim Vertrieb von Finanzinstrumenten kennt. Die Zulassung einer Zweigstelle darf von einem EU-Mitgliedstaat jedoch nur erteilt werden, wenn die Zweigstelle die Pflichten der Art. 16-20, 23-25, 27, 28 Abs. 1 und Art. 30-32 MiFID II sowie Art. 3-26 MiFIR und Art. 3-26 der VO (EU) Nr. 600/2014 einhält (Art. 41 Abs. 1 MiFID II). Zwar setzt Art. 41 Abs. 1 MiFID II nicht die Äquivalenz des Heimatsrechts der Zweigstelle mit dem der MiFID II voraus, sondern schreibt nur die Einhaltung der Regeln durch die Zweigstelle vor. Jedoch bewirkt die Regelung faktisch eine Rückwirkung auf die Schweiz, da der Gesetzgeber, der wegen der geschilderten Regelung der MiFIR die Gleichwertigkeit des Aufsichtsrechts bei der Erbringung von Leistungen an professionelle Kunden und geeignete Gegenparteien anstrebt, dann auch die Erbringung der Leistung an Retailkunden mitregeln kann. Da das schweizerische Aufsichtsrecht aufgrund der sektoriellen Aufsicht bislang keine vergleichbaren Regeln für alle Finanzintermediäre beim Vertrieb von Finanzinstrumenten kennt, schafft die Regelung des Art. 41 Abs. 1 MiFID II also einen weiteren Anreiz zu einer Regulierung durch den Schweizer Gesetzgeber.

Für eine Regelung spricht auch das Haftungsrisiko, das sich aus Art. 6 der Rom I-Verordnung und dem Verbrauchergerichtsstand von Art $15 \mathrm{ff}$. LugÜ ergeben kann, wenn mitgliedstaatliche Gerichte einzelnen MiFID IIRegelungen nicht nur anlegerschützenden, sondern auch verbraucherschützenden Charakter zumessen. Der in der Europäischen Union ansäs- 
sige Kunde kann dann als Verbraucher am Ort seines gewöhnlichen Aufenthalts gegen den schweizerischen Finanzintermediär klagen und sich auf die dort geltenden Verbraucherschutzbestimmungen berufen. Eine ganze Reihe von schweizerischen Instituten hat sich daher bereits heute dazu entschlossen, die Standards der Europäischen Union zu übernehmen, um drohende Haftungsansprüche zu vermeiden. Aus ihrer Sicht stellt eine Regelung der Wohlverhaltensregeln in der Schweiz daher keine zusätzliche Belastung dar.

Wenig überraschend sind grenzüberschreitend tätige Institute also im Grundsatz für eine Erweiterung des Aufsichtsrechts.5) Die rein inländischen Institute empfinden die Verschärfung dagegen als Überregulierung. ${ }^{6)}$ Dem hält die FINMA in ihrem 2010 veröffentlichten Bericht zu den Fällen Lehman und Madoff') zahlreiche Schwächen beim Vertrieb von Finanzinstrumenten entgegen, die den Gesetzgeber bewogen haben, die Wohlverhaltensregeln nicht nur für das grenzüberschreitende, sondern auch für das inländische Geschäft vorzuschreiben. Für diese Position spricht vor allem auch der Gesichtspunkt, dass sich bereits heute umfassende anlegerschützende Pflichten im Auftragsrecht finden und die Branche aufgrund der Selbstregulierung zahlreiche Wohlverhaltensregeln kennt. ${ }^{8)}$ Nicht alle der im geplanten Finanzdienstleistungsgesetz vorgeschlagenen Regelungen sind also neu. Vielmehr werden einige zivilrechtliche Verhaltenspflichten nun auch aufsichtsrechtlich geregelt (gleichsam "gedoppelt"), damit sie von der Aufsichtsbehörde durchgesetzt werden können, und andere - zuvor in der Selbstregulierung bereits vorhandene aufsichtsrechtliche Regelungen - werden auf die Gesetzesstufe angehoben. Mit

5) Vgl. zur Diskussion NZZ v. 17.1.2014, S. 31 („Privatbanken wollen Abkommen mit Brüssel“); NZZ v. 2.5.2014, S. 25 („Der Bundesrat soll auf Marktzutritt drängen Dringlicher Appell der Expertengruppe Brunetti zur Finanzplatzstrategie“); NZZ v. 3.6.2014, S. 25 („Sorgen um Schweizer Marktzugang in Europa"); NZZ v. 7.8.2014, S. 27 („Am fehlenden EU-Markt-Zugang ist die Branche selbst schuld“); NZZ v. 17.9.2014, S. 71 („Ein verletzliches Geschäft“).

6) NZZ v 4.4.2014, S. 27 („Eine Bedrohung für inlandorientierte Banken“).

7) FINMA, Madoff-Betrug und Vertrieb von Lehman-Produkten: Auswirkungen auf das Anlageberatungs- und Vermögensverwaltungsgeschäft, Bericht v. 2.3.2010.

8) Genannt sei nur SwissBanking, Verhaltensregeln für Effektenhändler bei der Durchführung des Effektenhandelsgeschäftes v. 22.10.2008 sowie VSV, Schweizerische Standesregeln für die Ausübung der unabhängigen Vermögensverwaltung v. 1.1.2014, beide für allgemeinverbindlich erklärt durch FINMA-Rundschreiben 2008/10 „Selbstregulierung als Mindeststandard“, Anhang N 10. 
diesem Vorgehen möchte die Schweiz ihr Finanzmarktrecht auf den international üblichen Regulierungsstandard bringen. ${ }^{9}$

Am 27. Juni 2014 schickte der Bundesrat den Vorentwurf für ein Finanzinstitutsgesetz (VE-FINIG) und ein Finanzdienstleistungsgesetz (VE-FIDLEG) in die Vernehmlassung. ${ }^{10)}$ Die eingegangenen Stellungnahmen wurden ausgewertet $^{11)}$ und flossen in die am 4. November 2015 publizierte Botschaft ein, ${ }^{12)}$ die voraussichtlich in der Frühjahrssession 2016 im Parlament behandelt wird.

\section{Der Geltungsbereich der Woblverbaltensregeln}

\section{Persönlicher Geltungsbereich}

Das FIDLEG ${ }^{13)}$ wird für alle Finanzdienstleister gelten (Art. 2 Abs. 1 lit. a). Hierunter sind alle juristischen oder natürlichen Personen zu verstehen, die gewerbsmäßig Finanzdienstleistungen in der Schweiz oder für Kundinnen und Kunden in der Schweiz erbringen (Art. 3 lit. e). ${ }^{14)}$ Dies sind zum einen bereits prudentiell beaufsichtigte Marktteilnehmer, wie Banken, Wertpapierhäuser, Fondsleitungen und Versicherungsunternehmen. Zum anderen werden alle Vermögensverwalter erfasst, die nach dem EFINIG ebenfalls einer prudentiellen Aufsicht durch eine separate Aufsichtsorganisation unterworfen werden (Art. 57 E-FINIG). Ist ein Finanzdienstleister eine natürliche Person, so hat er neben den Verhaltens- und Organisationspflichten auch die Vorschriften für Kundenberater einzuhalten (vgl. Art. 6, 30 f., 34 Abs. 2). ${ }^{15)}$ Reine Anlageberater unterstehen weiterhin keiner prudentiellen Aufsicht, wohl aber den Verhaltensregeln

9) Botschaft zum Finanzdienstleistungsgesetz (FIDLEG) und zum Finanzinstitutsgesetz (FINIG), v. 4.11.2015, BBl 2015, 8901, 8913, 8931.

10) https://www.admin.ch/ch/d/gg/pc/ind2014.html\#EFD.

11) Bericht des Eidgenössischen Finanzdepartements über die Vernehmlassungsergebnisse zum Finanzdienstleistungsgesetz (FIDLEG) und zum Finanzinstitutsgesetz (FINIG) v. 13.3.2015, http://www.news.admin.ch/NSBSubscriber/message/attachments/38717.pdf.

12) Vgl. Botschaft zum Finanzdienstleistungsgesetz (FIDLEG) und zum Finanzinstitutsgesetz (FINIG), v. 4.11.2015, BBl 2015, 8901, 8913, 8931.

13) Alle nachfolgend zitierten Gesetzesbestimmung sind solche des E-FIDLEG, sofern nicht anders vermerkt.

14) Dem Gesetz nicht unterstellt sind die Schweizerische Nationalbank (SNB) und die Bank für Internationalen Zahlungsausgleich (BIZ), vgl. Art. 2 Abs. 2 E-FIDLEG.

15) Vgl. Botschaft zum Finanzdienstleistungsgesetz (FIDLEG) und zum Finanzinstitutsgesetz (FINIG), v. 4.11.2015, BBl 2015, 8901, $8947 \mathrm{f}$. 
des FIDLEG. Um auch bei ihnen die Durchsetzung dieser Pflichten sicherzustellen, finden sich entsprechende Strafbestimmungen (Art. 92 ff.).

Erfasst sind weiterhin Kundenberater (Art. 2 Abs. 1 lit. b). Hierunter sind alle natürlichen Personen zu verstehen, die selbst oder im Namen eines Finanzdienstleisters Finanzdienstleistungen an Kunden erbringen (Art. 3 lit. f). Gemäß der Botschaft ist der Begriff „Kundenberater“ weit auszulegen. Insbesondere fallen neben Anlageberatern und Vermögensverwaltern auch Versicherungsvermittler und Vertriebsträger darunter. ${ }^{16)}$ Das FIDLEG geht also funktional vor und erfasst als Kundenberater alle Personen, die am Point of Sale gegenüber Kunden auftreten. ${ }^{17)}$

Kundenberater bedürfen einer Registrierung im Beraterregister, wenn sie für einen in- oder ausländischen Finanzdienstleister tätig sind, der selbst über keine Zulassung nach Art. 3 FINMAG verfügt (Art. 30), z. B. in- oder ausländische Anlageberater. Die Registrierung darf nur erfolgen, wenn sie

(1) eine entsprechende Aus- und Weiterbildung belegen können,

(2) eine Berufshaftpflichtversicherung oder gleichwertige Sicherheiten nachweisen und

(3) sie einer Ombudsstelle angeschlossen sind.

(4) Ausgeschlossen ist die Registrierung bei Vorhandensein von bestimmten Vorstrafen und

(5) bei Vorliegen eines aufsichtsrechtlich verhängten Berufsverbots (Art. 31).

Sie bedürfen dann keiner eigenen Berufshaftpflicht oder gleichwertiger Sicherheit und keines Anschlusses an eine Ombudsstelle, wenn sie nachweisen, dass ihr Arbeitgeber diese Voraussetzungen erfüllt (Art. 31 Abs. 3, Abs. 1 lit. c). Das Register ist öffentlich (Art. 34 Abs. 5).

Als Angestellte oder als Vertreter tätige Kundenberater von in der Schweiz zugelassenen Finanzdienstleistern müssen sich dagegen nicht re-

16) Vgl. Botschaft zum Finanzdienstleistungsgesetz (FIDLEG) und zum Finanzinstitutsgesetz (FINIG), v. 4.11.2015, BBl 2015, 8901, $8922 \mathrm{f}$.

17) Personen, die die Erbringung von Finanzdienstleistungen nur in untergeordneter Weise unterstützen (z. B. durch Versand von Produktdokumentationen, Vereinbarung eines Termins für einen Kunden bei seinem Kundenberater etc.) gelten nicht als Kundenberater, Botschaft zum Finanzdienstleistungsgesetz (FIDLEG) und zum Finanzinstitutsgesetz (FINIG), v. 4.11.2015, BBl 2015, 8901, 8948. 
gistrieren lassen. ${ }^{18)}$ Bei ihnen stellt der Finanzdienstleister ihre fachliche Eignung sicher (Art. 7, 24, 25).

Schließlich unterfallen auch Ersteller und Anbieter von Finanzinstrumenten dem Gesetz (Art. 2 Abs. 1 lit. c), auf die aus Platzgründen nicht näher eingegangen wird.

\section{Sachlicher Geltungsbereich}

Das FIDLEG erfasst Finanzdienstleistungen, worunter es folgende Tätigkeiten versteht: den Erwerb oder die Veräußerung von Finanzinstrumenten, die Annahme und Übermittlung von Aufträgen, die Finanzinstrumente zum Gegenstand haben, die Anlageberatung in Finanzinstrumenten sowie die Gewährung von Krediten für die Durchführung von Geschäften mit Finanzinstrumenten (Art. 3 lit. d). Auch die individuelle Vermögensverwaltung ist erfasst. Im Gegensatz zum deutschen Recht bezieht sich dieser Tatbestand nicht nur auf die Verwaltung von Finanzinstrumenten, sondern auch auf diejenige von Finanzanlagen (vgl. Art. 3 lit. a i. V. m. lit. d Ziff. 3). Hierunter sind etwa Bankguthaben auf Sicht oder auf Zeit und Forderungspapiere ohne Effektencharakter zu verstehen. Nicht als Finanzanlagen gelten dagegen direkte Anlagen in Immobilien und Ansprüche aus Sozialversicherungen sowie Guthaben der beruflichen Vorsorge. ${ }^{19)}$ Der Tatbestand der Vermögensverwaltung unterscheidet sich noch in einer weiteren Hinsicht von dem des deutschen Aufsichtsrechts. Im FIDLEG werden nicht nur die auf Dauer mittels Vollmacht getätigten Vermögensverwaltungen erfasst, sondern auch Vollmachten, die sich auf einzelne Geschäfte beziehen, und Verhältnisse, bei denen Transaktionen trotz externer Vollmachten intern vom Kunden zu genehmigen sind, ${ }^{20)}$ so dass im Innenverhältnis faktisch eine Anlageberatung vorliegt. Aus Sicht des Aufsichtsrechts soll jedoch das Außenverhältnis für die Einordnung in die Bewilligungstatbestände maßgeblich sein.

Weit fällt im Vergleich zum Anhang I Abschnitt C der MiFID II der Begriff der Finanzinstrumente aus (vgl. Art. 3 lit. b Ziff. 6, 7), der auch

18) Botschaft zum Finanzdienstleistungsgesetz (FIDLEG) und zum Finanzinstitutsgesetz (FINIG), v. 4.11.2015, BBl 2015, 8901, 8967.

19) So ausdrücklich die Botschaft zum Finanzdienstleistungsgesetz (FIDLEG) und zum Finanzinstitutsgesetz (FINIG), v. 4.11.2015, BBl 2015, 8901, 8943.

20) Botschaft zum Finanzdienstleistungsgesetz (FIDLEG) und zum Finanzinstitutsgesetz (FINIG), v. 4.11.2015, BBl 2015, 8901, 8946. 
rückkaufsfähige Lebensversicherungen mit kursabhängigen Leistungen und Abfindungswerten erfasst, weil bei diesen der Kunde ein Kapitalanlagerisiko trägt. ${ }^{21}$ Als Finanzinstrumente gelten auch Kapitalisations- und Tontinengeschäfte sowie Einlagen, deren Rückzahlungswert oder Zins risiko- oder kursabhängig ist, es sei denn, deren Zins ist an einen Zinsindex gekoppelt.

\section{Räumlicher Geltungsbereich}

Schweizer Finanzdienstleister unterstehen dem FIDLEG unabhängig davon, ob sie Dienstleistungen für Kunden in der Schweiz oder im Ausland erbringen. Ausländische Finanzdienstleister werden vom Gesetz erfasst, wenn sie sich an Kunden in der Schweiz wenden. Anders als beim räumlichen Anwendungsbereich des BankG kommt es also nicht auf in der Schweiz errichtete Zweigniederlassungen oder die Bestellung eines Vertreters in der Schweiz an (Art. 2 BankG, Art. 2 ABV-FINMA). Grenzüberschreitende Bankgeschäfte ohne dauerhafte physische Präsenz dürfen somit erlaubnisfrei erbracht werden. ${ }^{22)}$ Dass das FIDLEG nun den räumlichen Anwendungsbereich ausweitet, wird mit der Schutzbedürftigkeit des inländischen Publikums begründet; ausländische Finanzdienstleister sollen die gleichen Verhaltensregeln einhalten müssen wie Schweizer Anbieter, um inländische Kunden gleich gut zu schützen. Der Gesetzgeber macht allerdings zu Recht die Einschränkung, dass die Durchsetzung der Vorschriften gegenüber ausländischen Finanzdienstleistern wegen des Territorialitätsprinzips nur schwer möglich sein wird. ${ }^{23)}$ In Deutschland wird diese Lücke zumindest zum Teil durch die Rechtsprechung geschlossen, die dem Kunden bei ohne Zulassung erbrachten Bankgeschäften und Finanzdienstleistungen einen Schadensersatzanspruch zubilligt, wobei sie den Schaden im Eingehen der Vertragsbeziehung sieht. ${ }^{24)}$ Am Beispiel der Vermögensverwaltung zeigt sich, dass dem Kunden hierdurch eine PutOption gewährt wird. Hat sein Vermögensverwalter gut gewirtschaftet, beendet der Kunde die Vertragsbeziehung, wenn er von der fehlenden Zulassung erfährt. Hat er schlecht gewirtschaftet, kann und wird der

21) Botschaft zum Finanzdienstleistungsgesetz (FIDLEG) und zum Finanzinstitutsgesetz (FINIG), v. 4.11.2015, BBl 2015, 8901, 8943.

22) Bahar/Stupp in: Basler Komm-BankG, 2. Aufl. 2013, Art. 1 Rz. 85.

23) Botschaft zum Finanzdienstleistungsgesetz (FIDLEG) und zum Finanzinstitutsgesetz (FINIG), v. 4.11.2015, BBl 2015, 8901, $8916 \mathrm{f}$.

24) Vgl. etwa BGH, Urt. v. 11.7.2006 - VI ZR 340/04, WM 2006, 1896 ff. 
Kunde verlangen, so gestellt zu werden, als habe er von dem Vermögensverwalter nie gehört, so dass er als Schadensersatz die Differenz des Werts des heutigen Portfolios zum ursprünglich eingesetzten Kapital erhält. ${ }^{25)}$ Entsprechende Urteile deutscher Gerichte sind gemäss Art. 33 ff. LugÜ II anzuerkennen und können gemäss Art. 38 ff. LugÜ in der Schweiz vollstreckt werden ${ }^{26)}$ Eine vergleichbare abschreckende Rechtsprechung fehlt in der Schweiz.

\section{Die Kundenkategorien}

Das Schweizer Recht kennt Kundenkategorien bereits im Kollektivanlagerecht. Der Gesetzgeber führt nun im FIDLEG die drei aus der MiFID bekannten Kundenkategorien ein (Art. 4, 5), übernimmt sie aber nicht vollständig. Ein erster Unterschied besteht darin, dass man öffentlichrechtliche Körperschaften, Vorsorgeeinrichtungen und Einrichtungen der beruflichen Vorsorge sowie Unternehmen nicht pauschal als geborene professionelle Kunden einordnet, sondern nur, wenn sie über eine professionelle Tresorerie verfügen. Dies ist der Fall, wenn sie über mindestens eine fachlich ausgewiesene, im Finanzbereich erfahrene Person verfügen, die ihre Finanzmittel dauernd bewirtschaftet. ${ }^{27)}$ Dieses Kriterium, das an das vorhandene Know-how anknüpft, erscheint dem Gesetzgeber überzeugender als das mit der MiFID II vorgenommene pauschale Abstellen auf Unternehmensgröße oder Status einer Organisation. Der zweite Unterschied findet sich bei der Möglichkeit zum Wechsel der Kundengruppe. Gemäß Art. 5 Abs. 1 können vermögende Privatkunden erklären, dass sie als professionelle Kunden gelten wollen (sog. Opting-out). Der Bundesrat kann die Eignung dieser Personen als professionelle Kunden zusätzlich von Voraussetzungen, namentlich von fachlichen Qualifikationen, abhängig machen. Es ist geplant, die Regelung in Art. $6 \mathrm{KKV}$ zu übernehmen. ${ }^{28)}$ Damit könnten Privatkunden, die aufgrund ihrer Ausbildung und beruflichen Erfahrung oder aufgrund einer vergleichbaren Erfahrung im Finanzsektor über die Kenntnisse verfügen, die notwendig sind, um die Risiken der Anlagen zu verstehen, und die über ein Vermögen von mindestens

25) BGH, Urt. v. 9.11.2010 - VI ZR 303/09, WM 2011, 17 ff.; OLG Köln, Urt. v. 16.1.2013 - 16 U 29/12, IPRspr 2013, Nr. 173, 374 ff.

26) BGer, Urt. v. 29.12.2008-4A_440/2008; BGer, Urt. v. 5.10.2009-4A_305/2009.

27) FINMA-RS 2008/5 Erläuterungen zum Begriff Effektenhändler, Rz. $1 \overline{6}$.

28) Botschaft zum Finanzdienstleistungsgesetz (FIDLEG) und zum Finanzinstitutsgesetz (FINIG), v. 4.11.2015, BBl 2015, 8901, 8949 f. 
500.000 Fr. verfügen, zu professionellen Kunden hochgestuft werden. Sofern diese Lösung in der geplanten Verordnung umgesetzt würde, entspräche sie derjenigen der MiFID II. Art. $6 \mathrm{KKV}$ geht jedoch noch darüber hinaus und erlaubt auch Personen, die schriftlich bestätigen, über ein Vermögen von mindestens 5 Mio. Fr. zu verfügen, eine Höherstufung. Damit wäre das Schweizer Recht deutlich weniger paternalistisch als das entsprechende europäische. Auf der anderen Seite ist jedoch zu bedenken, dass Geld allein noch keine Erfahrung ersetzt. ${ }^{29)}$

\section{Die Interessenwahrungspflicht}

\section{Eignungs- und Angemessenheitstest}

Gemäß Art. 8 Abs. 2 hat der Finanzdienstleister die Interessen seiner Kunden zu wahren. Diese Regelung ist ein Beispiel für die Doppelung der im Zivilrecht bereits vorhandenen Interessenwahrungs- bzw. Treuepflicht (Art. 398 Abs. 2 OR) mit dem Ziel, ihre aufsichtsrechtliche Durchsetzung zu ermöglichen. Ausfluss dieser Pflicht ist der Eignungstest. Aus diesem Grund verpflichtet Art. 13 Vermögensverwalter dazu, die Geeignetheit der von ihm getätigten Anlagen für den Kunden zu prüfen. Dazu muss er sich bei Privatkunden nach deren finanziellen Verhältnissen, Anlagezielen sowie Kenntnissen und Erfahrungen erkundigen. Bei professionellen Kunden reicht die Frage nach den Anlagezielen (Art. 15) ${ }^{30} .{ }^{31)}$ Leider ist die Gesetzesbegründung sehr widersprüchlich, da sie zu Art. 14 feststellt:

„Da Finanzdienstleister bei professionellen Kunden regelmässig auf die Durchführung einer Angemessenheitsprüfung verzichten können (vgl. Art. 15), beziehen sich die Einschränkungen von Artikel 14 vorab nur auf Dienstleistungen für Privatkundinnen und -kunden. ${ }^{(32)}$

29) Vgl. bereits FINMA, Regulierung von Produktion und Vertrieb von Finanzprodukten an Privatkunden - Stand, Mängel und Handlungsoptionen („FINMA-Vertriebsbericht 2010“) vom Oktober 2010, S. 41; ablehnend daher Sethe, SJZ 2014, 477, 484; Sethe, SZW 2014, 615, 625.

30) Botschaft zum Finanzdienstleistungsgesetz (FIDLEG) und zum Finanzinstitutsgesetz (FINIG), v. 4.11.2015, BBl 2015, 8901, $8958 \mathrm{f}$.

31) Es sei denn, dass der Finanzdienstleister Anhaltspunkte dafür hat, dass der Kunde die Risiken des Geschäfts nicht versteht, Botschaft zum Finanzdienstleistungsgesetz (FIDLEG) und zum Finanzinstitutsgesetz (FINIG), v. 4.11.2015, BBl 2015, 8901, 8958.

32) Botschaft zum Finanzdienstleistungsgesetz (FIDLEG) und zum Finanzinstitutsgesetz (FINIG), v. 4.11.2015, BBl 2015, 8901, 8958. 
In der Begründung zu Art. 15 heißt es dann zutreffend:

„Die Vorschriften der Angemessenheits- und Eignungsprüfung gemäss Artikel 12 und 13 gelten grundsätzlich auch bei Finanzdienstleistungen für professionelle Kunden. Allerdings ist bei deren Durchführung den besonderen Eigenschaften dieses Kundensegments Rechnung zu tragen. Der Finanzdienstleister muss deshalb nur dann einen Abgleich des Geschäfts mit den Erfahrungen und Kenntnissen der Kundinnen und Kunden vornehmen, wenn Anhaltspunkte bestehen, die Zweifel an einem ausreichenden Verständnis des Geschäfts hervorrufen. ${ }^{\text {“'33) }}$

Gerade die zweite zitierte Passage macht deutlich, dass bei professionellen Kunden nur die Exploration der Kenntnisse und Erfahrungen und der mit der Finanzdienstleistung einhergehenden finanziellen Tragbarkeit entfällt, nicht aber die Berücksichtigung der Anlageziele. Alles andere wäre absurd, weil Art. 15 ansonsten dem Vermögensverwalter erlauben würde, bei professionellen Kunden deren Vermögen ohne Rücksicht auf die Anlageziele anzulegen. Der Wortlaut der Erkundigungspflicht in Art. 13 ist im Übrigen enger als Art. 25 Abs. 2 MiFID II, da er die Verlusttragungsfähigkeit und die Risikotoleranz nicht erwähnt. Auf die entsprechende Kritik $^{34)}$ hat der Bundesrat reagiert und führt in der Botschaft aus, dass das Risikobewusstsein, die Risikofähigkeit und -bereitschaft zu erfragen sind. ${ }^{35)}$

Auch bei der Anlageberatung besteht eine inhaltlich entsprechende Pflicht zur Geeignetheitsprüfung. Allerdings schränkt der Gesetzgeber diese Prüfung auf Fälle ein, in denen der Anlageberater das gesamte Portfolio des Kunden kennt (Art. 13). Wünscht der Kunde dagegen nur eine Anlageberatung für eine einzige Transaktion, ohne dem Anlageberater sein übriges Portfolio aufzudecken, schuldet der Finanzintermediär lediglich einen Angemessenheitstest (Art. 12). Damit unterscheidet sich die Regelung deutlich von Art. 25 Abs. 2 MiFID II, der den Geeignetheitstest für jede Form der Anlageberatung vorschreibt. Der weniger intensive Angemessenheitstest ist in Art. 25 Abs. 3 MiFID II für die beratungsfreien Leistungen (reines Ausführungsgeschäft) reserviert und kann gemäß Art. 25 Abs. 4 MiFID II für nichtkomplexe Finanzinstrumente ganz abbedungen werden.

33) Botschaft zum Finanzdienstleistungsgesetz (FIDLEG) und zum Finanzinstitutsgesetz (FINIG), v. 4.11.2015, BBl 2015, 8901, 8958.

34) Kritisch daher bereits Weber/Sethe, Äquivalenz als Regelungskriterium im Finanzmarktrecht, SJZ 2014, 569, 577; Sethe, SZW 2014, 615, 625; gemäß dem Bericht des Eidgenössischen Finanzdepartements über die Vernehmlassungsergebnisse zum Finanzdienstleistungsgesetz (FIDLEG) und zum Finanzinstitutsgesetz (FINIG) v. 13.3.2015, S. 19, forderte auch Lenz \& Staehelin eine diesbezügliche Erweiterung des Gesetzeswortlauts.

35) Botschaft zum Finanzdienstleistungsgesetz (FIDLEG) und zum Finanzinstitutsgesetz (FINIG), v. 4.11.2015, BBl 2015, 8901, 8957 f. 
Das FIDLEG sieht dagegen beim reinen Ausführungsgeschäft sogar ganz vom Angemessenheitstest ab (Art. 14) und zwar sowohl bei nichtkomplexen als auch bei komplexen Finanzinstrumenten. ${ }^{36)}$ Gleiches gilt in Bezug auf Leistungen, die auf Veranlassung des Kunden erbracht werden.

\section{Organisatorische Pflichten zur Interessenwabrung}

Diese Verhaltenspflicht wird in Art. 27 durch organisatorische Pflichten, in Art. 28 durch eine Regelung zu Retrozessionen und in Art. 29 durch Vorgaben zu Mitarbeitergeschäften ergänzt. Bemerkenswert ist der Wandel zwischen dem Vorentwurf und der Botschaft in Bezug auf den immanenten Interessenkonflikt zwischen der Vergütung des Finanzdienstleisters einerseits und dem Interesse des Kunden an einer von Vergütungsinteressen unbeeinflussten Anlage seines Vermögens andererseits. Der Vorentwurf sah in Anlehnung an MiFID II noch die Unterscheidung zwischen auf Provisionsbasis und auf Honorarbasis arbeitenden Finanzdienstleistern vor (Art. 9 VE-FIDLEG), wobei diese Regelung sowohl für Anlageberater als auch für Vermögensverwalter gelten sollte. Dafür war - abweichend von Art. 24 Abs. 8 MiFID II - Vermögensverwaltern weiterhin die Annahme von Retrozessionen erlaubt, sofern sie diese entweder an die Kunden weiterleiteten oder der Kunde auf deren Herausgabe verzichtete (dazu sogleich). Die Unterscheidung zwischen auf Provisionsbasis und auf Honorarbasis arbeitenden Finanzdienstleistern ist in der Botschaft ersatzlos entfallen.

Eine Änderung gab es auch hinsichtlich der Zulässigkeit der Annahme von Retrozessionen. Im Vorentwurf war vorgesehen, dass Finanzdienstleister im Zusammenhang mit der Erbringung von Finanzdienstleistungen nur Vorteile annehmen dürfen, wenn die Kunden vorgängig ausdrücklich auf die Herausgabe der Vorteile verzichtet haben oder die Vorteile vollumfänglich an die Kunden weitergegeben werden (Art. 26 Abs. 1 VEFIDLEG). Diese Lösung, die nicht nur die Frage der Information, sondern auch die rein zivilrechtliche Folgefrage der Herausgabe der Retrozession ansprach, ging über die Vorgaben von Art. 24 Abs. 9 MiFID II hinaus und stieß deshalb als aufsichtsrechtliche Überregulierung auf viel Kritik, so dass der Bundesrat in der Botschaft zurückrudern musste. Nun

36) Kritisch zum Vorentwurf daher die Stellungnahme des Verbandes der Auslandsbanken in der Schweiz v. 17.9.2014, S. 14, http://www.foreignbanks.ch/vas.exe?Modul=DO\& Dokument =5055; kritisch auch Weber/Sethe, SJZ 2014, 569, 577. 
ist in Art. 28 Abs. 1 und 2 vorgesehen, dass Finanzdienstleister im Zusammenhang mit der Erbringung von Finanzdienstleistungen von Dritten Entschädigungen annehmen dürfen, wenn sie die Kunden vorgängig ausdrücklich über die Entschädigung informiert haben oder die Entschädigung vollumfänglich an die Kunden weitergeben. Die vorgängige Information der Kunden muss Art und Umfang der Entschädigung beinhalten bzw. die Berechnungsparameter und Bandbreiten, wenn Art und Umfang noch nicht genau feststehen. Das Schweizer Recht folgt damit dem Ansatz von MiFID I und entscheidet sich bewusst gegen die Modifikation, die MiFID II (zwei Arten der Anlageberatung und Totalverbot der Retrozessionen bei Vermögensverwaltern) mit sich bringt. Man darf gespannt sein, ob die Europäische Union diese Lösung als äquivalent anerkennt. ${ }^{37)}$

\section{Fazit}

Das vorgelegte Reformpaket aus FINIG und FIDLEG, dessen Begründung in der Botschaft 192 Seiten umfasst, kann an dieser Stelle nicht umfassend gewürdigt werden. Eines seiner Hauptanliegen, die unabhängigen Vermögensverwalter einer Aufsicht zu unterwerfen, ist unter Anlegerschutzgründen ebenso zu begrüßen wie unter Wettbewerbsgründen, da bislang nur ein Teil der diese Leistung anbietenden Intermediäre beaufsichtigt wird.

Gelungen ist auch die Verpflichtung zur Aus- und Weiterbildung der am Point of Sale tätigen Personen. Positiv einzuordnen ist zudem die umfassende Geltung des FIDLEG für in- und ausländische Finanzdienstleister, soweit sie sich an Kunden in der Schweiz wenden. Allerdings ist die Durchsetzung der Regulierung gegenüber ausländischen Anbietern schwach ausgeformt.

Auf den ersten Blick kritisch zu sehen ist die geplante Einordnung von Anlegern als professionelle Kunden allein wegen ihres großen Vermögens, ohne dass es auf ihre Erfahrung oder Vorkenntnisse im Wertpapiergeschäft ankäme. Im Ergebnis wird diese Lösung, die bereits in Art. $6 \mathrm{KKV}$ für die Kollektivanlagen verankert ist, allerdings dadurch entschärft, dass der Finanzintermediär/der unabhängige Vermögensverwalter die Anleger über ihren Status als qualifizierte Anleger informieren, sie über die damit einhergehenden Risiken aufklären und sie auf die Möglichkeit hinweisen

37) Zweifelnd Sethe, SJZ 2014, 477, 484 ff.; auf den Unterschied zu Art. 24 Abs. 8 MiFID II aufmerksam macht auch Bärtschi, Finanzmarktregulierung im Fluss, SZW 2014, 459, 481. 
muss, schriftlich erklären zu können, nicht als qualifizierte Anleger gelten zu wollen (Art. 6a Abs. 2 lit. c KKV). Somit kann der Anleger auf wohlinformierter Grundlage eine Entscheidung treffen, während ihm MiFID II diese Autonomie versagt.

Als überzeugend erweist sich die Einordnung öffentlich-rechtlicher Körperschaften, Vorsorgeeinrichtungen, Einrichtungen der beruflichen Vorsorge und Unternehmen in die Kategorie der professionellen Kunden, die nur erfolgt, sofern sie über eine professionelle Tresorerie verfügen. Hieran sollte sich der europäische Gesetzgeber ein Beispiel nehmen, da im Gegensatz zur Regelung in der MiFID II nicht auf den Status, sondern auf das vorhandene Fachwissen abgestellt wird.

Die Regelung zur Eignungsprüfung bei der Vermögensverwaltung ist als Fortschritt zu werten, da die Aufsicht nun diesen im Auftragsrecht anerkannten Test überwachen und durchsetzen kann. Warum der Gesetzgeber sich aber bei der Anlageberatung dazu entschlossen hat, die Eignungsprüfung nur vorzuschreiben, wenn der Anlageberater das Gesamtportfolio des Kunden kennt, erschließt sich mir nicht. Denn auch bei der Anlageberatung in Bezug auf Einzelwerte ohne Kenntnis des Beraters vom Gesamtportfolio des Kunden ist eine Eignungsprüfung möglich, allerdings eben nur in Bezug auf das eine Finanzinstrument. Wenn der Kunde diese eingeschränkte Perspektive wünscht, heißt das jedoch noch nicht, dass man ihm den Schutz der Eignungsprüfung auch im Übrigen versagen müsste. Kauft der Kunde ein einzelnes Finanzinstrument, das erkennbar nicht zu seinen finanziellen Verhältnissen passt (z. B. wegen seiner Laufzeit), ist der Berater nun gemäß Art. 12 gerade nicht verpflichtet, den Kunden darüber in Kenntnis zu setzen, geschweige denn zu warnen. Das überzeugt nicht.

Verwundert ist man im Übrigen, dass im Bereich des reinen Ausführungsgeschäfts jeglicher Test, und damit eine Warnpflicht des Finanzdienstleisters bei erkennbar unpassenden Anlagen durch den Kunden, fehlt. Damit bleibt das FIDLEG hinter der schweizerischen Rechtsprechung zurück: Führt ein Finanzintermediär für einen Kunden ein Einzelgeschäft aus, ist er grundsätzlich nicht zu einer generellen Interessenwahrung verpflichtet; eine Aufklärungspflicht besteht daher nur auf Verlangen oder bei Vorliegen besonderer Umstände. ${ }^{38)}$ Solche Umstände bestehen bei offensichtlich erkennbarer Aufklärungsbedürftigkeit des Kunden oder bei Bestehen eines

38) BGer, Urt. v. 15.1.2002 - 4C.265/2001, E. 2.a; BGE 119 II 333, 335; BGE 133 III 98, 103 E. 7.1.2. 
besonderen Vertrauensverhältnisses zwischen Finanzintermediär und Kunde, aus welchem Letzterer nach Treu und Glauben auch unaufgefordert Beratung und Abmahnung erwarten durfte. ${ }^{39)}$ Dies ist in der Sache nichts anderes als der Angemessenheitstest, bei dem der Kunde zu warnen ist, wenn er erkennbar (!) seinen bisherigen Anlagehorizont verlässt und die Risiken nicht einschätzen kann. Zudem stellt sich auch hier die Frage, ob die Europäische Union dies als äquivalent ansehen wird.

Schließlich fallen die Unterschiede zwischen MiFID und FIDLEG bei der Bewältigung des Problems der Entlohnung des Finanzintermediärs auf. Das FIDLEG schließt sich der Lösung von MiFID I an. Dass man die Rechtsfigur des Honorarberaters nicht übernommen hat, erscheint verständlich, vermag sich dieser doch bislang auch in vielen Mitgliedstaaten der Europäischen Union noch nicht am Markt durchsetzen. Nachdenklicher stimmt es jedoch, dass der Gesetzgeber die besonderen Gefahren von Retrozessionen in der Vermögensverwaltung in der Botschaft nicht ausreichend adressiert. Anders als bei der Anlageberatung trifft der Kunde des Vermögensverwalters gerade nicht im Vorhinein Entscheidungen über Anlageobjekte in Kenntnis der Kosten und der Art und der Höhe der Retrozessionen. Vielmehr stimmt er zu Beginn der Vermögensverwaltung abstrakt der Annahme von Retrozessionen zu und erfährt im Nachhinein aus den Rechenschaftsberichten, welche Geschäfte getätigt wurden und welche Retrozessionen geflossen sind, ohne aber zu wissen, ob andere Anlageobjekte vielleicht geringere Retrozessionen ausgelöst hätten und aus diesem Grund für den Vermögensverwalter als Anlageobjekt ausgeschieden sind. Der Anleger hat also ein Kontrollproblem, das im FIDLEG ungelöst bleibt. Man wird sicherlich in der Schweiz, aber auch in der Europäischen Union, mit großem Interesse die langfristigen Erfahrungen von Mitgliedstaaten beobachten, die Retrozessionen ganz verboten haben (wie etwa Großbritannien mit dem "Retail Distribution Review"). ${ }^{40}$

39) BGE 133 III 97, 102 E. 7.1.1; BGE 119 II 333, 335 E. 5a; BGer, Urt. v. 15.1.2002 4C.265/2001, E. 2.a; BGer, Urt. v. 23.7.2002 - 4C.108/2002, E. 2.b (= Pra 92 [2003], Nr. 51); BGer, Urt. v. 23.6.1998 - 4C.410/1997, E. 3.b. (= Pra 77 [1998] Nr. 155); BGE 124 III 162; BGE 119 II 333, 335; vgl. zum Ganzen Eggen, Finanzprodukte, Auftrag oder Kauf?, SZW 2011, 625, 629; Thalmann, Von der vorvertraglichen Aufklärungspflicht der Bank zur börsengesetzlichen Informationspflicht des Effektenhändlers, in: FS für Druey, 2002, S. 973 ff.; Thalmann, Die Sorgfaltspflicht der Bank im Privatrecht insbesondere im Anlagegeschäft, ZSR 1994 II, 229 ff.

40) Vgl. dazu die Hinweise unter https://www.fca.org.uk/news/post-implementation-reviewof-the-rdr. 